\title{
Accelerated evolution at chaperone promoters among Antarctic notothenioid fishes
}

Samuel N. Bogan ${ }^{1,2^{*}}$ (D) and Sean P. Place

\begin{abstract}
Background: Antarctic fishes of the Notothenioidei suborder constitutively upregulate multiple inducible chaperones, a highly derived adaptation that preserves proteostasis in extreme cold, and represent a system for studying the evolution of gene frontloading. We screened for Hsfl-binding sites, as Hsfl is a master transcription factor of the heat shock response, and highly-conserved non-coding elements within proximal promoters of chaperone genes across 10 Antarctic notothens, 2 subpolar notothens, and 17 perciform fishes. We employed phylogenetic models of molecular evolution to determine whether (i) changes in motifs associated with Hsf1binding and/or (ii) relaxed purifying selection or exaptation at ancestral cis-regulatory elements coincided with the evolution of chaperone frontloading in Antarctic notothens.

Results: Antarctic notothens exhibited significantly fewer Hsf1-binding sites per bp at chaperone promoters than subpolar notothens and Serranoidei, the most closely-related suborder to Notothenioidei included in this study. 90\% of chaperone promoters exhibited accelerated substitution rates among Antarctic notothens relative to other perciformes. The proportion of bases undergoing accelerated evolution (i) was significantly greater in Antarctic notothens than in subpolar notothens and Perciformes in 70\% of chaperone genes and (ii) increased among bases that were more conserved among perciformes. Lastly, we detected evidence of relaxed purifying selection and exaptation acting on ancestrally conserved cis-regulatory elements in the Antarctic notothen lineage and its major branches.

Conclusion: A large degree of turnover has occurred in Notothenioidei at chaperone promoter regions that are conserved among perciform fishes following adaptation to the cooling of the Southern Ocean. Additionally, derived reductions in Hsf1-binding site frequency suggest cis-regulatory modifications to the classical heat shock response. Of note, turnover events within chaperone promoters were less frequent in the ancestral node of Antarctic notothens relative to younger Antarctic lineages. This suggests that cis-regulatory divergence at chaperone promoters may be greater between Antarctic notothen lineages than between subpolar and Antarctic clades. These findings demonstrate that strong selective forces have acted upon cis-regulatory elements of chaperone genes among Antarctic notothens.
\end{abstract}

Keywords: Comparative genomics, Heat shock proteins, Notothen, Promoters, cis-regulation, Gene regulation, Environmental adaptation

\footnotetext{
* Correspondence: snbogan@ucsb.edu

${ }^{1}$ Department of Biology, Sonoma State University, Rohnert Park, CA 94928,

USA

2Department of Ecology, Evolution and Marine Biology, University of

California, Santa Barbara, CA 93106, USA
}

(c) The Author(s). 2019 Open Access This article is distributed under the terms of the Creative Commons Attribution 4.0 International License (http://creativecommons.org/licenses/by/4.0/), which permits unrestricted use, distribution, and reproduction in any medium, provided you give appropriate credit to the original author(s) and the source, provide a link to the Creative Commons license, and indicate if changes were made. The Creative Commons Public Domain Dedication waiver (http://creativecommons.org/publicdomain/zero/1.0/) applies to the data made available in this article, unless otherwise stated. 


\section{Background}

It is widely supported that selection upon cis-regulatory networks drives the rapid turnover of transcription factor binding sites across evolution [1-5] and can subsequently give rise to the emergence or alteration of complex traits $[6,7]$. Among eukaryotes, selection often acts upon conserved cis-regulatory elements (CREs) as a function of the evolutionary lineage at which they arose. For example, the exaptation or overprinting of ancestral enhancer regions is a common phenomenon in the evolution of cis-regulatory novelties [8-13]. Numerous variations to this pattern exist in nature however, indicating that precise modes of cisregulatory evolution are more complex than is currently understood [14, 15]. Pathway-specific investigations of selection at CREs founded in the evolution of distinctive and derived traits, particularly in non-model systems, can provide valuable insight into the diversity of evolutionary mechanisms acting on gene regulatory networks in nature $[16,17]$.

Changes in the induction or basal expression level of transcripts are hallmarks of gene regulatory evolution in animals. In ectotherms for example, increases in the basal expression of stress-inducible genes that confer a derived, constitutive function are evident in many cases of physiological adaptation to environmental limits. These "frontloaded" genes are often hampered in their capacity for induction and arise among intraspecific groups occupying novel environments [18-29] or among interspecific groups [30-34]. The evolutionary mechanisms that drive inducible genes toward constitutive states are also important in understanding oncogenesis [35-37], pathogen resistance to drugs [38-40], and the evolution of functional morphology [41, 42].

The gene regulatory mechanisms conferring frontloaded states of expression for classes of genes remain unexplored. This is, in part, due to challenges in determining whether a gene's regulation is evolutionarily fixed or environmentally regulated. A stark example of evolved frontloading is the constitutive upregulation of inducible chaperones (i.e. heat shock proteins or HSPs) among Antarctic fishes of the perciform suborder Notothenioidei, a trait that convergently arose in Cryonototheniodei (Antarctic notothens) and other Antarctic eukaryotes including invertebrates and ciliates during adaptation to the progressive cooling and stenothermy of the Southern Ocean [4346]. Antarctic notothens constitutively express inducible chaperones to mitigate constant cold denaturation to their proteome [47] and multiple Antarctic lineages are unable to further upregulate chaperones in response to heightened stress [48, 49] while some Antarctic and secondarilysubpolar notothens still exhibit classical HSP induction $[50,51]$.

Constitutive HSP expression has a clear advantage under chronic cold. Investigations of chaperone demand and chaperone activity in Notothenioidei suggest this trait is fixed and unresponsive to increases or decreases in chaperone demand in at least some species [52-54]. As the rapid upregulation of HSPs via interactions with $H s f 1$, the master transcription factor of the classical heat shock response, is a conserved regulatory trait among almost all eukaryotes [55], subpolar and Antarctic notothens offer a valuable comparative system for exploring divergence in cis-regulatory homology as it relates to the evolution of novel gene regulatory states.

The classical heat shock response is one of the best studied models of inducible gene expression and is crucial in the adaptation of ectotherms across environmental gradients [56]. Recently, a diversity of mechanisms have been uncovered that are associated with or link variation in molecular chaperone function to environmental adaptation via transposable element architecture, DNA methylation, copy number variation, and coding sequence divergence [57]. To our knowledge, variation in the cis-regulatory homology of heat shock genes has been explored in three studies in an evolutionary context. Two of these studies examined differences in the inducibility and constitutive expression of different molecular chaperones and found that inter- or intraspecific groups varied in their capacity for induction but not constitutive expression levels [58-60]. Exploring divergence in cis-regulatory regions of notothen heat shock genes across the subantarctic-to-Antarctic transition and subsequent radiation may (i) offer insights into mechanisms promoting gene frontloading and (ii) contribute to our growing understanding of cis-regulatory evolution.

To this end, we identified Hsf1-binding site motifs or heat shock elements (HSEs) of the consensus sequence GAAnnTTCnnGAA and highly-conserved CREs within the promoters of HSP7O and HSP9O chaperone genes from 32 species of fishes representing 10 cryonotothens, 2 subpolar notothens, 17 perciformes, and 3 actinopterygian outgroups. We employed phylogenetic models of mutation rate and conservation to (i) test whether frequencies of HSEs vary among Cryonotothenioidei, subpolar notothens, and other perciformes at chaperone promoters and (ii) determine whether CREs that are highly-conserved among perciformes and/or subpolar notothens exhibit relaxed purifying selection or exaptation in Cryonotothenioidei, which would signify cisregulatory divergence from ancestrally-conserved features of chaperone promoters.

\section{Results}

\section{Extraction and alignment of orthologous promoter} regions

From here forward, we use gene names for chaperone CDS that were drawn from the Notothenia coriiceps genome assembly [51] in our reporting of this study. 
From a total of 16 full-length CDS for HSP7O and HSP90 genes annotated in notothenioid genome assemblies, we were able to extract orthologous promoter regions from a majority of notothen and non-notothen assemblies for 10 genes (7 HSP70; 3 HSP90). Chaperone promoter regions were represented by an average coverage of 29.4 species $\pm 2.25 \mathrm{SD}$. The average length of multiple sequence alignments of promoters across all examined species was $2.95 \mathrm{~kb} \pm 1.45 \mathrm{SD}$. Multiple alignments of orthologous promoters from Notothenioidei alone averaged $2.55 \mathrm{~kb}$ in length $\pm 1.15 \mathrm{SD}$. Accessions and links to genome assemblies and chaperone CDS are available in Additional file 1.

\section{HSE motif frequency in chaperone promoters across Perciformes and Notothenioidei}

In order to examine potential changes in cis-regulatory homology specific to Hsf1 signaling, we measured the number of canonical HSE motifs within proximal promoter regions upstream of chaperone CDS. Accounting for phylogeny, Antarctic notothens exhibited significantly fewer HSE motifs $\mathrm{bp}^{-1}$ than subpolar notothens (Fig. 1; $\mathrm{F}=7.75 ; p=0.009$ ). Effect size estimates for the reduction in cryonotothen HSEs relative to subpolar outgroups were large (Cliff's delta $=-1.0$ ) while the wide distribution of HSEs $\mathrm{bp}^{-1}$ in non-notothen fishes resulted in a negligible effect size (Cliff's delta $=-.064$ ). This small difference in HSE frequency $\mathrm{bp}^{-1}$ between cryonotothens and non-notothen fishes was primarily driven by high HSE frequencies within the three nonperciform outgroup species. Comparing cryonotothens to non-notothen perciforms demonstrated no significant difference in HSE frequency $\mathrm{bp}^{-1}$. Rather than reducing the density of HSEs within chaperone promoters relative to a perciform ancestral state, cryonotothens exhibited fewer HSEs $\mathrm{bp}^{-1}$ relative to subpolar notothens and the
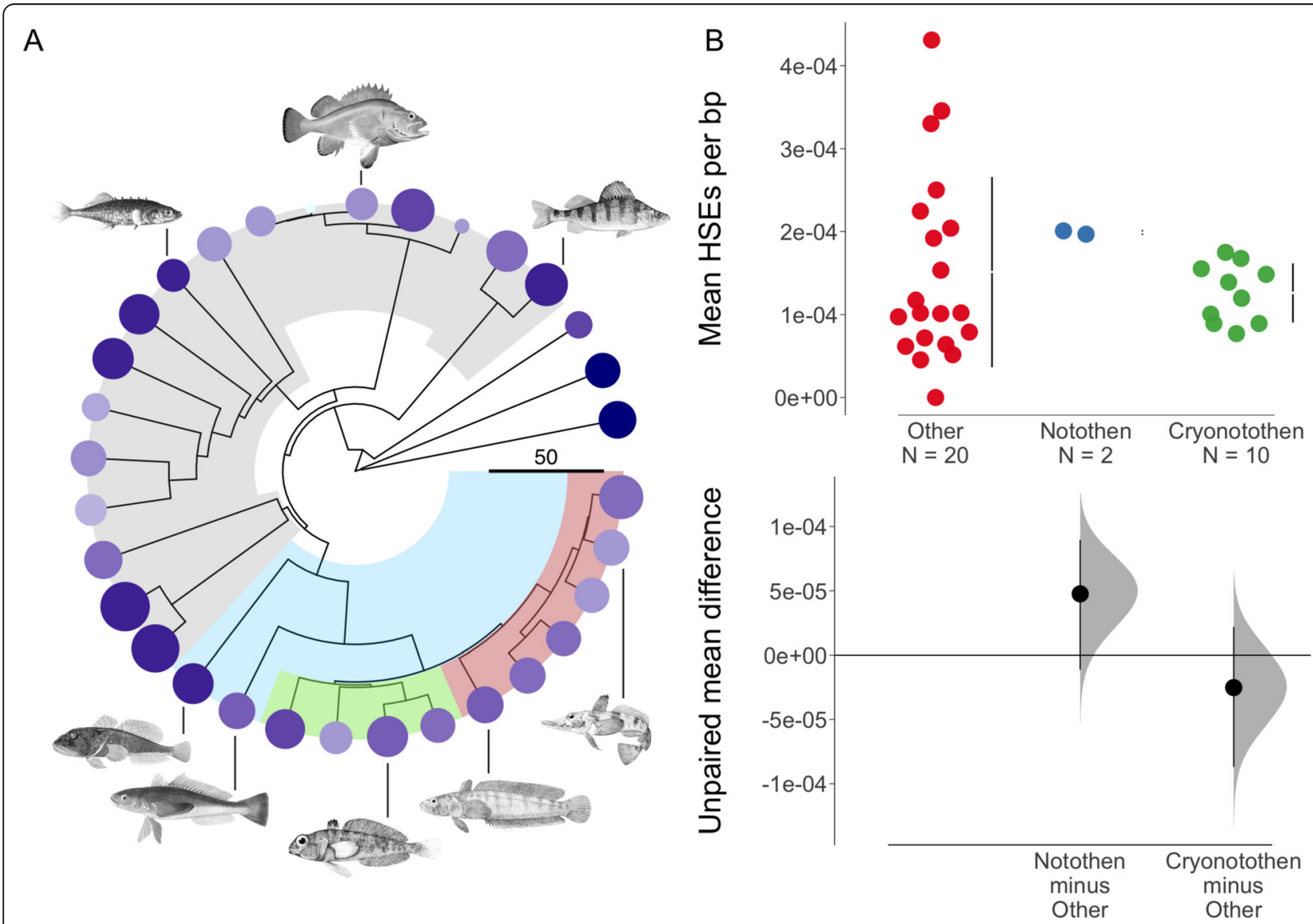

Fig. 1 Changes in HSE frequency bp ${ }^{-1}$ at chaperone promoters across Perciformes. a A time-calibrated phylogeny of Perciform fishes drawn from Chang et al. 2019, including Nototheniodei and three non-perciform outgroups, is visualized. The scale key depicts a 50-million-year span. Nodes shaded in grey represent Perciform suborders, except in the case of Notothenioidei which is shaded in blue. The two major lineages of Cryonototheniodei are depicted in red and green. Circles at branch tips are colored according to the mean frequency of HSE motifs detected among chaperone promoters for a given species (light = low frequency; dark = high frequency). Circle sizes are scaled to the summed lengths of promoter sequences analyzed. All illustrations of fishes are public domain. $\mathbf{b}$ Bootstrapped effect size estimates for variation in HSE frequency bp' among cryonotothens (Cryonotothen), subpolar notothens (Notothen) and other perciformes and outgroups (Other) 
nearest non-notothen suborder, Serranoidei (Fig. 1a; Cliff's delta $=-.64)$.

As expected, the average number of HSE motifs per promoter varied across chaperone genes and species. The mean frequency of HSE motifs for each gene, averaged across all fishes, ranged from 0.0-1.4. The median HSE frequency equaled 0.15. The mean coefficient of variation (CV) for HSE frequency equaled $2.42 \pm 2.17 \mathrm{SD}$ when mean HSE frequency was sampled across all species for a given gene. At finer evolutionary scales, this variation was reduced. The mean CV for HSE frequency among notothens equaled $0.85 \pm 1.28$ SD. Lastly, we found that HSE motifs were poorly conserved across the perciform phylogeny. Across 54 promoter regions predicted to be highly-conserved among perciformes by the phastCons [61] algorithm, only 1 conserved region contained a consensus for the canonical HSE motif. Mean HSE frequencies and densities averaged across genes are listed for each species in Additional file 2.

\section{Accelerated mutation rates at chaperone promoters of Antarctic notothens}

In order to test for accelerated mutation rates in cryonotothen chaperone promoters against highly-conserved ancestral CREs, which could be tied to relaxed purifying selection or the positive and negative selection of elements with high turnover, we (i) measured the mutation rates of whole promoters across evolutionary time within the cryonotothen lineage relative to subpolar notothens, perciformes, and three non-perciform actinopterygians and (ii) examined the proportion of accelerated bases relative to conserved and neutrally constrained bases in each chaperone promoter among these clades. We found that 9 out of 10 chaperone genes exhibited significantly accelerated mutation rates in Cryonotothenioidei relative to the rest of the examined phylogeny $(p<0.0001)$.

The single gene that did not exhibit an accelerated rate of promoter mutation among cryonotothens was HSP90A. In contrast, the $H S P 90 A$ promoter was significantly more conserved among cryonotothens than orthologous promoter regions in all other examined species $(p<0.0001)$. Comparing phyloP conservation/acceleration scores expressed as - $\log p$-values averaged across bases demonstrated that HSP9OA was the only chaperone to exhibit a positive score (Cliff's delta $=0.39$ ), indicating that the majority of the HSP90A promoter showed stronger patterns of conservation as opposed to acceleration (Fig. 2; Additional file 3).

We also quantified the proportion of bases within chaperone promoters under significant acceleration in order to determine (i) the extent to which increased rates of mutation within Cryonototheniodei were evident across these genomic features and (ii) compare the proportion of accelerated bases across Antarctic notothens, subpolar notothens, and other perciform fishes. In 7 out of

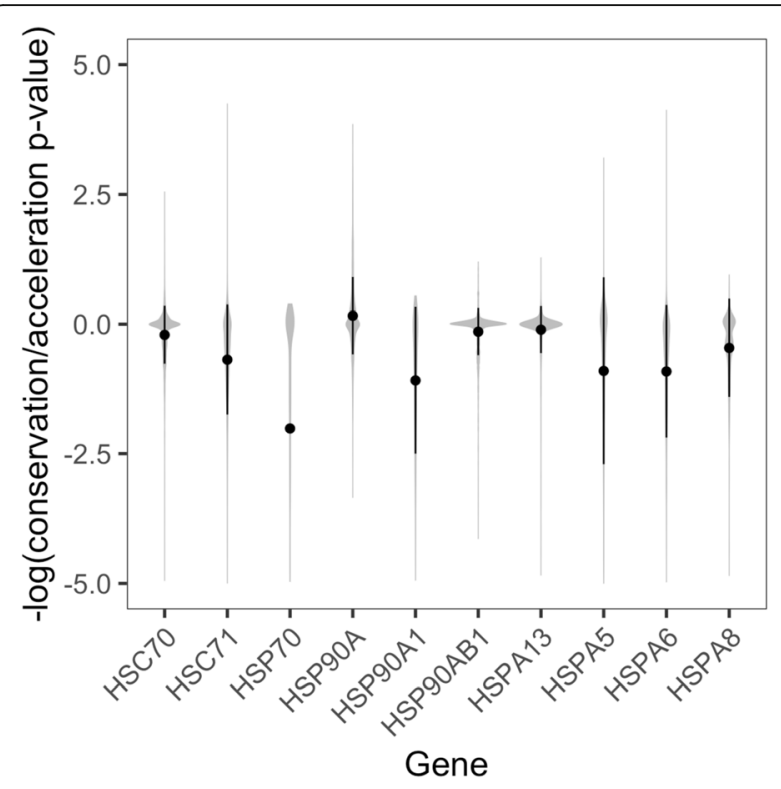

Fig. 2 Base-by-base conservation and acceleration scores among cryonotothen chaperone promoters. Signed -log $p$-values for the likelihood of heightened selection on discrete base pairs within Cryonototheniodei relative to other perciformes are plotted for conservation (positive value) and acceleration (negative value) across each chaperone promoter. Circles depict means. Error bars depict \pm SD

10 genes, cryonotothens exhibited a significantly greater proportion of accelerated bases compared to both subpolar notothens and other perciforms (Fig. 3). The three genes for which this was not true were HSC70, HSP90A, and HSP9OAB1. Additionally, these three genes and HSP70 exhibited significantly lower proportions of accelerated bases relative to conserved bases (as opposed to acceleration relative to all bases) in cryonotothens compared to subpolar notothens and Perciformes (Fig. 3; $F=4.01-27.35$; $p \leq 0.0001-0.0181)$. In summary, a large majority of chaperone genes exhibited accelerated evolution at their promoters within the cryonotothen lineage in terms of (i) mutation rate across whole promoters and (ii) the proportion of promoter bases that had undergone significant accelerated evolution.

\section{Turnover of highly-conserved ancestral CREs among Antarctic notothens}

We sought to determine how patterns of acceleration varied between poorly conserved promoter regions and highly-conserved putative regulatory elements. After calculating the conservation of bases across each chaperone promoter among all species included in this study, we found that more conserved promoter regions held a significantly higher proportion of bases exhibiting significant acceleration in Cryonotothenioidei (Fig. 4; $x^{2}=$ 34.56; $p<0.0001$ ). Additionally, this positive relationship did not significantly vary between chaperones from 


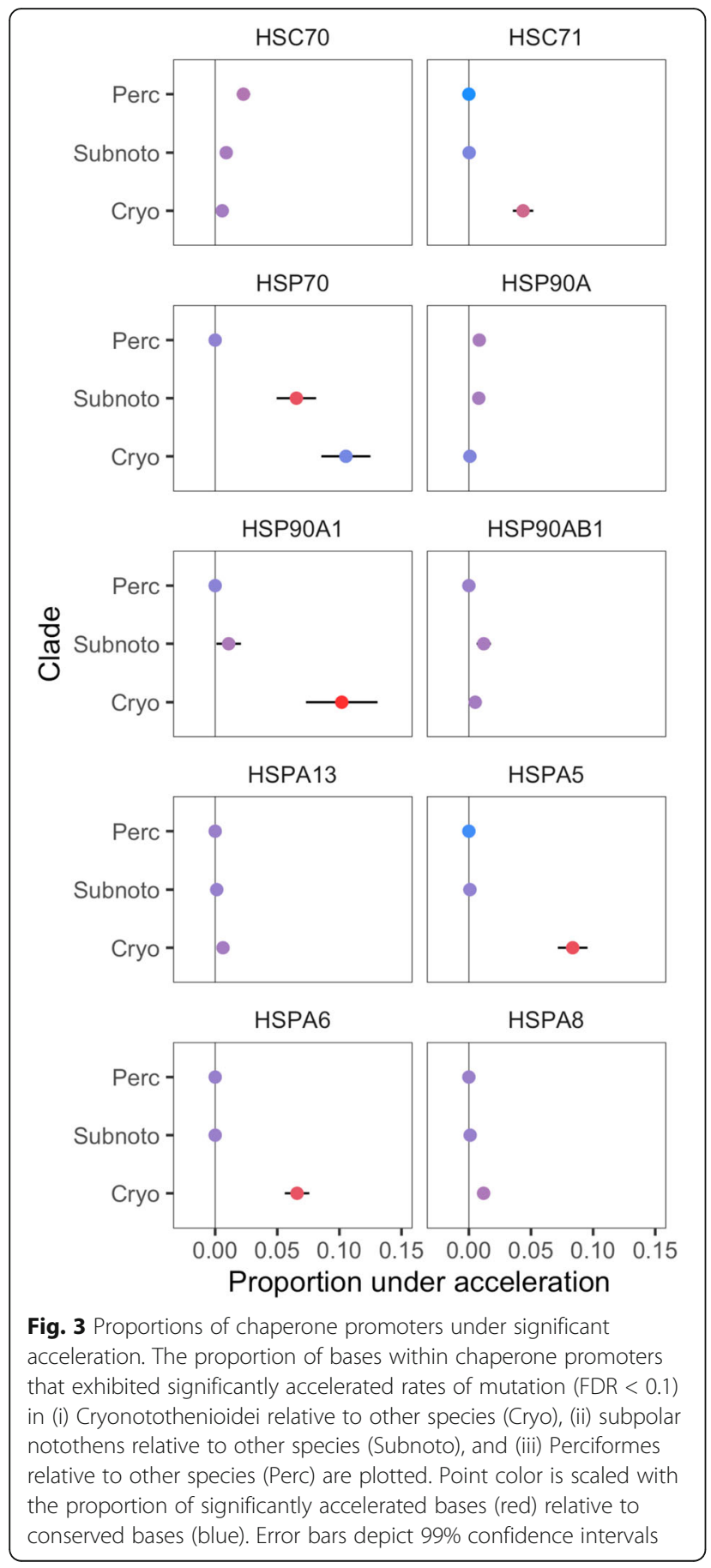

HSP70 and HSP9O gene families (Fig. 4; $\mathrm{x}^{2}=2.31 ; p=$ 0.1281). Thus, increased rates of mutation within cryonotothen chaperone promoters were enriched among highlyand ancestrally-conserved bases, suggesting relaxed purifying selection at these sites.

In order to more robustly test for patterns of relaxed purifying selection at ancestral cis-regulatory regions, we also tested for accelerated rates of mutation among cryonotothens within conserved putative CREs

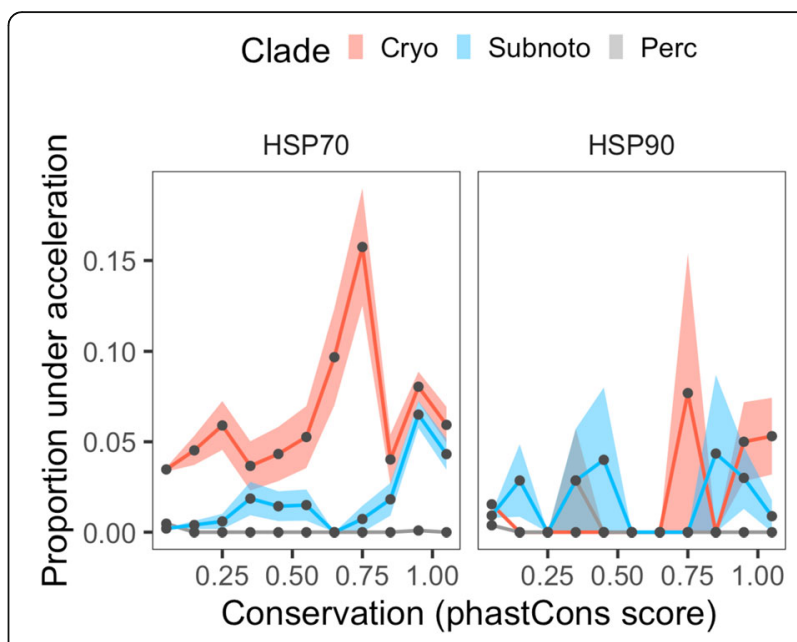

Fig. 4 Changes in the proportion of accelerated bases as a function of promoter conservation. The proportions of bases within

chaperone promoters that exhibited significantly accelerated rates of mutation (FDR < 0.1) in Cryonotothenioidei (Cryo), subpolar notothens (Subnoto), and Perciformes (Perc) are plotted for HSP7O and HSP9O chaperone genes against binned phastCons scores representing conservation across the 32-species phylogeny employed in this study. Shaded regions depict \pm SE

identified in chaperone promoters by the phastCons algorithm [61, 62]. Base-by-base phastCons scores are available in Additional file 4. Additionally, we also tested for losses of ancestrally-conserved elements and lineagespecific gains of new putative elements within Notothenioidei using dless [61]. Gains of lineage-specific promoter elements among cryonotothens would suggest that accelerated promoter evolution is, in part, tied to the evolution of novel features.

We detected 15 instances of significantly accelerated rates of mutation in Cryonotothenioidei at CREs highlyconserved among Perciformes. These accelerated CREs were distributed across 4 chaperone genes. Two accelerated CREs, both within the HSP90A promoter, were accelerated within one of the two major cryonotothen clades. Only one accelerated CRE overlapped with a promoter region that underwent a lineage-specific gain-offunction within Cryonotothenioidei (Fig. 5) demonstrating that acceleration at these ancestrally-conserved CREs was largely due to relaxed purifying selection or negative selection rather than gains of function.

Despite evidence of relaxed purifying selection and/or negative selection at CREs conserved among Perciformes, cryonotothen chaperone promoters exhibited more lineage-specific gains of CREs than losses. Additionally, discrete lineage-specific gains were predicted to span a larger proportion of promoters than losses. CRE gains occurred widely across all promoter regions and overprinted on ancestrally-conserved CREs in 9 out of 18 instances. Only 3 gains were predicted to have evolved at 


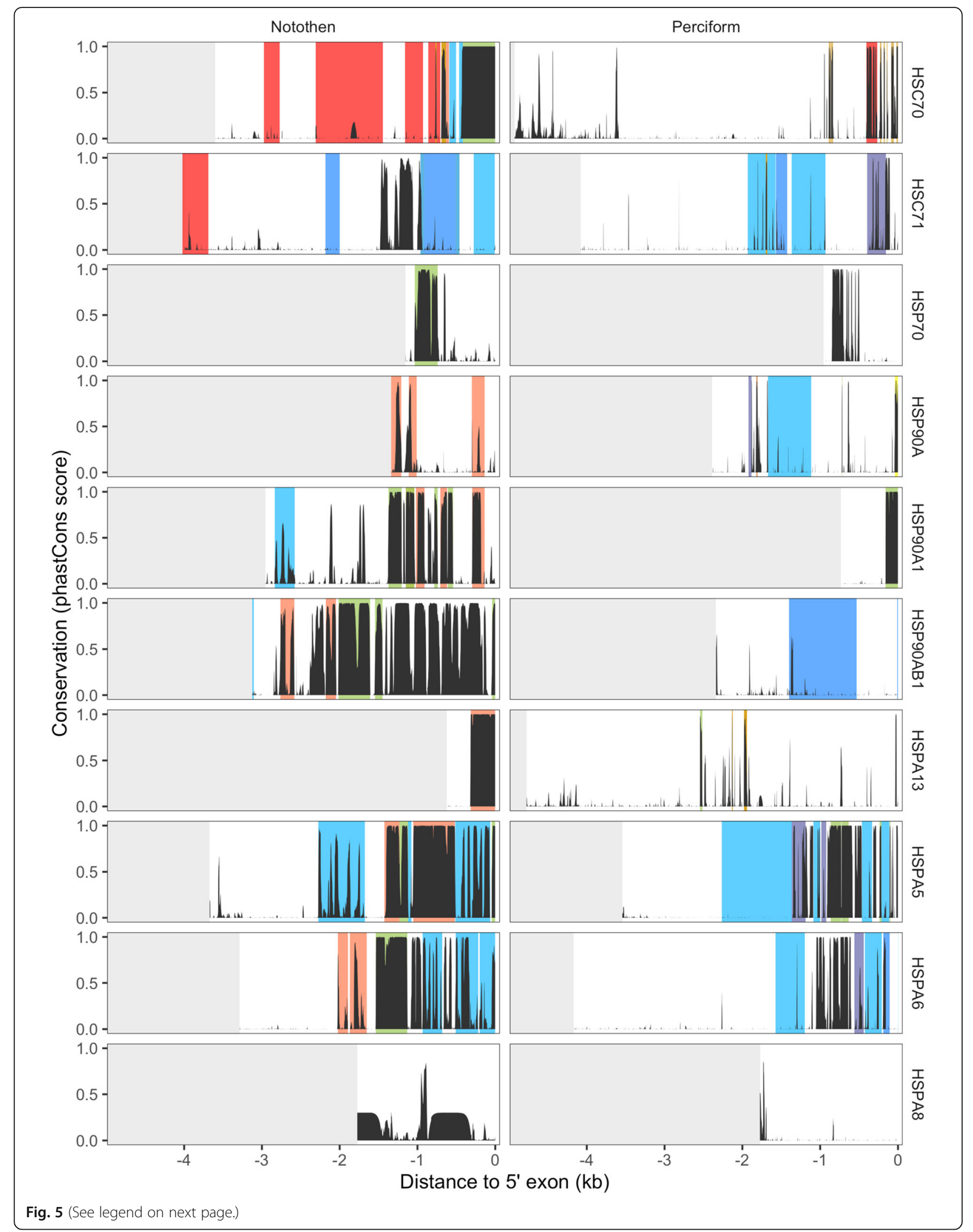


(See figure on previous page.)

Fig. 5 CRE turnover events at chaperone promoters within Notothenioidei. phastCons scores estimated for all 32 species examined in this study (Perciform) and the Notothenioidei subset of this phylogeny (Notothen) are plotted across 5' $-3^{\prime}$ promoter ends. Grey annotations depict the absence of data. Orange annotations depict significantly accelerated rates of mutation within Cryonotothenioidei and yellow annotations depict the same for younger cryonotothen lineages. Blue bars depict CRE gains in either subpolar notothens (dark blue), Cryonototheniodei (blue) or younger cryonotothen lineages (light blue). Red annotations depict CRE losses in either Cryonotothenioidei (dark red) or younger cryonotothen lineages (light red). Green annotations depict significant CRE conservation in Cryonotothenioidei relative to other species

the Cryonotothenioidei node, while 10 gains were predicted to have occurred at younger cryonotothen lineages and 2 among subpolar notothen nodes. By contrast, only one CRE exhibiting high ancestral conservation among Perciformes appeared to have undergone a complete functional loss at the ancestral Cryonotothenioidei node. Testing for accelerated or functionally lost CREs that were conserved among Notothenioidei in exclusion of conservation among other Perciformes revealed numerous losses across multiple lineages of Cryonotothenioidei (Fig. 5). The evolution of chaperone promoters across Cryonotothenioidei appears to be a composite of relaxed purifying selection and overprinting at highlyconserved ancestral CREs, with the multitude of CRE losses and gains having occurred in Antarctic branches that evolved after Cryonotothenioidei.

\section{Discussion}

\section{Reduced HSE frequencies among cryonotothen chaperone promoters}

Measuring the number of canonical Hsf1-binding motifs or HSEs in HSP9O and HSP7O chaperone promoters among cryonotothens, subpolar notothens, and other perciformes and actinopterygians revealed that Antarctic notothens exhibited fewer HSEs $\mathrm{bp}^{-1}$ than subpolar notothens and Serranoidei, the nearest suborder to Notothenioidei included in this study. Furthermore, HSE motifs were poorly conserved across the perciform phylogeny and were highly variable in their frequency within a given gene's promoter even among closely-related species.

Taken together, these two findings suggest a pattern of purifying selection against canonical HSEs within the promoters of some HSP9O and HSP70 chaperones during the adaptive radiation of Cryonotothenioidei across the Southern Ocean. Mutagenesis studies and comparative work in natural populations have demonstrated that losses of canonical HSEs within chaperone promoters can result in or is associated with diminished induction of downstream expression [60, 63, 64]. Many Antarctic notothens exhibit reduced or absent induction of the classical heat shock response. Therefore, the functional consequences of reductions in canonical HSEs at HSP9O and HSP7O genes should be explored among notothens in greater detail using functional genomic methods such as anti-Hsf1 ChIP-seq or broader assays for cis-regulatory activity such as ATAC-seq in tandem with transcriptomic profiling following heat stress in subpolar and Antarctic lineages.

\section{Relaxed purifying selection in Cryonotothenioidei at conserved CREs}

The dynamics of evolutionary changes in gene regulatory elements and their subsequent effects on gene expression can be shaped, in part, by relaxed purifying selection acting on conserved non-coding elements [65] and has been demonstrated in at least one adaptive radiation [56]. Ultimately, the proportion of relaxed purifying selection occurring at conserved non-coding regions relative to the rest of the non-coding genome can vary [66]. The majority of chaperone genes analyzed in this study demonstrate (i) accelerated evolution across promoters in Antarctic notothens relative to subpolar notothens and Perciformes and (ii) exhibit a greater proportion of accelerated bases across whole promoters in Antarctic notothens than in subpolar notothens and Perciformes. Understanding how these patterns of selection have shaped ancestral or derived promoter elements is therefore of great interest. Among Cryonotothens, which demonstrate highly derived constitutive levels of inducible chaperones, accelerated evolution consistent with relaxed purifying selection or negative selection at promoter elements that are highly-conserved among perciform fishes is not ubiquitous across chaperone genes and is only evident in 3 genes despite occurring at $27.8 \%$ of CREs (Fig. 5). We conclude that relaxed purifying selection or negative selection against promoter elements that are deeply-conserved among perciformes may not have a generalizable association with derived patterns of constitutive chaperone expression by the Antarctic cryonotothen lineage.

Among highly-conserved CREs identified in the Notothenioidei suborder in exclusion of conservation among other perciformes, $47.7 \%$ demonstrated either accelerated evolution or functional loss within the cryonotothen lineage, but not necessarily at the ancestral node of Cryonotothenioidei. Only 1/3 of instances of acceleration or functional loss at conserved elements occurred at the ancestral cryonotothen node. The remainder were detected in younger cryonotothen lineages. While constitutive chaperone expression is ubiquitous across 
Antarctic notothens, the ability of different species to upregulate inducible chaperones appears to vary across the two major cryonotothen lineages $[48,49,51]$. The extent to which lineage-specific variation in promoter homology across cryonotothen clades may help explain divergent patterns of chaperone induction should be further explored.

\section{Exaptation in Cryonotothenioidei at conserved CREs}

Exaptation, defined here as the overprinting of new elements on conserved ancestral non-coding regions, is a key phenomenon in the evolution of cis-regulatory novelty [ 8 , $10,11,13,67,68]$. This finding has largely been documented either in taxa spanning wide evolutionary distances $[12,69-71]$ or in clades that have speciated at normal rates $[7,67]$. Few studies have examined cis-regulatory evolution across adaptive species radiations such as the rapid diversification of Antarctic notothens. Adaptive radiations offer a unique insight into processes of molecular evolution given past evidence of accelerated evolution at coding regions during rapid diversifications $[17,72]$. Similar studies focusing on accelerated evolution in the non-coding regulatory regions of adaptively significant genes are scarce and have not yet demonstrated whether exaptation is important across the selective landscape acting on gene regulatory elements during such radiations.

In this study, we demonstrated that the evolution of novel promoter elements in Cryonotothenioidei was more frequent than functional losses of CREs conserved across Perciformes. Furthermore, $70 \%$ of new promoter elements that arose within Cryonotothenioidei chaperone promoters evolved at positions that contained highly-conserved CREs, not accounting for three instances of exaptation that occurred at subpolar nodes of Notothenioidei, demonstrating pervasive exaptation of ancestral promoter elements. Similar to patterns of relaxed purifying selection against highly-conserved CREs described above, instances of exaptation were more common among younger lineages of Cryonotothenioidei rather than the ancestral cryonotothen node (Fig. 5). While our study did detect strong signals of accelerated evolution across whole chaperone promoters as well as instances of relaxed purifying selection or exaptation at conserved promoter elements in Cryonotothenioidei, it is clear these instances were more pervasive in diverging Antarctic lineages. This finding is interesting in relation to genome-wide evidence of heightened genomic diversification at the ancestral subpolar node of Cryonotothenioidei and Eleginops maclovinus, the closest sister species to cryonotothens and a species included in this study [73], which suggests that the successful radiation of Notothenioidei within the Southern Ocean may have been shaped more by historical contingency than adaptive evolution [74]. In the case of chaperone genes, we found a greater degree of evidence that adaptive evolution has shaped lineage-specific variation in chaperone cis-regulatory homology within Cryonotothenioidei lineages as opposed to subpolar nodes ancestral to Cryonotothenioidei.

\section{Conclusion}

Environments characterized by frequent or chronic stress can select for the constitutive frontloading of gene families that are classically stress-inducible [19-34]. In systems for which constitutive expression of inducible genes is evolved, rather than plastic, it is not yet understood how natural selection has acted upon gene regulatory mechanisms to derive constitutive states. These systems are valuable for understanding the diversity of mechanisms contributing to gene regulatory evolution.

The heat shock response pathway of Antarctic notothens appears to have incurred a loss of canonical $H s f 1$ binding sites (i.e. HSEs) among HSP70 and HSP90 promoters coinciding with the evolution of constitutive expression among classically-inducible chaperones. The poor conservation of HSE motifs across Perciformes suggests that changes in the density and architecture of Hsfl-binding sites may be frequent and highly variable, potentially contributing to the reduction in HSEs $\mathrm{bp}^{-1}$ among Antarctic notothens via reduced constraint and turnover.

Lastly, accelerated rates of mutation were evident at chaperone promoters among Antarctic notothens relative to subpolar notothens and other perciformes. It appears that heightened selection acted upon the majority of HSP70 and HSP9O chaperone promoter regions during adaptation to the cooling of the Southern Ocean. Accelerated mutation rates were predominantly explained by instances of relaxed purifying selection or exaptation at highly-conserved promoter elements that were lineage specific to more recently-evolved cryonotothen branches. While our study did uncover derived cis-regulatory modifications to molecular chaperone genes that evolved at the ancestral Cryonotothenioidei node, it also demonstrated that differential selection acting on proximal CREs distinct from $H_{s f 1}$ signaling may be stronger between different cryonotothen lineages than between subpolar and Antarctic lineages. These results add to a small but growing body of work regarding the regulatory origins of constitutive chaperone expression by Antarctic notothens and loss of the inducible heat shock response [38, 92, 93]. More broadly, our findings help shed light on processes contributing to cis-regulatory evolution in genes underpinning environmental adaptation and subsequent adaptive radiations.

\section{Methods}

Multiple sequence alignment of proximal chaperone promoter regions

Proximal promoter regions were identified for HSP7O and HSP9O paralogs by aligning full coding sequences or 
CDS of chaperone genes annotated in the Notothenia coriiceps genome assembly [51] to genome assemblies for 31 other fishes including 10 cryonotothens [75-78], 2 subpolar notothens [76, 79], 20 perciform fishes [79] and three non-perciform outgroups [79-81] using BLASTn [82] and extracting all $5 \mathrm{~kb}$ DNA upstream of the 1st exon of the highest fidelity CDS mapping to $N$. coriiceps query sequence. When $N$. coriiceps CDS did not successfully align to the first exon of a given ortholog, a CDS identified in an annotation from the nearest relative was used. Chaperone CDS that did not successfully align to orthologous 1st exons of at least $75 \%$ of species included in this study were not included in downstream analyses. A list of all species and their corresponding assemblies as well as a list of chaperone CDS annotations in notothen genomes are included in Additional file 1.

$N$. coriiceps chaperone CDS were used if they were (i) present in annotations for genome assemblies of the notothens Notothenia coriiceps and Cottoperca gobio and (ii) mapped to RNA-seq transcripts in both species as demonstrated in the NCBI RefSeq database. $5 \mathrm{~kb}$ regions upstream of 1st exons were also mapped against the NCBI nucleotide collection using BLASTn [82]. Any portion of query sequences that aligned to $>5 \%$ of a CDS from another gene were trimmed from the 3 ' end of that region to the $5^{\prime}$ end of the query sequence in order to ensure that only non-coding DNA was present in promoter sequences. This approach maintained regions of the $5^{\prime}$ UTR of chaperone genes within analyzed promoter regions. However, CDS encoding 5' UTRs play a significant role in cisregulation and were thus kept [83]. In $1.9 \%$ of orthologous promoters analyzed across all genes and species, chaperone CDS were unable to be mapped to the 1st exon of a given species' ortholog, but a majority of the corresponding promoter was identified via alignment to a promoter from a nearby species. Multiple alignments of orthologous promoter regions were generated using Clustal Omega [84].

\section{Motif screening for HSE motifs and phylogenetic comparisons of HSE density}

HSE motifs matching the consensus sequence GAAnnTTCnnGAA and its complement were identified by querying the motif file MA0486.2, a motif derived from consensus ChIP-seq Hsf1 binding sites in Homo sapiens, against trimmed promoter sequences across all species and their orthologous chaperone genes using FIMO [85] and counting the frequency of motif matches that (i) strictly fit the consensus HSE motif and (ii) were scored with a Benjamini-Hochberg adjusted $p$-value of $<0.05$. The frequency of HSE motifs per $\mathrm{bp}^{-1}$ within each promoter region was then calculated.

Resulting values were used to test whether cryonotothens exhibited significant differences in HSE frequency relative to subpolar notothens and other outgroups using a phylogenetically-corrected ANOVA run with the 'phylANOVA' function in the phytools $\mathrm{R}$ package [86], v0.6.99, set to 1000 simulations and a Holm-Bonferroni $p$-value adjustment. The phylogenetic topography applied to phylANOVA as well as all other phylogenetic analyses was drawn from the 'fish tree of life' multi-locus phylogeny of actinopterygians interfaced in the fishtree $R$ package [87], v0.3.1. Cliff's delta effect sizes for variation in HSE frequency between clades were estimated using the 'cliff' function in the effsize $\mathrm{R}$ package [88], v0.7.1. This package was used for all other Cliff's delta estimates performed in this study. Mean effect sizes were visualized for Fig. 1 using the R package dabestr [89], v0.2.2.

\section{Measuring accelerated evolution at whole promoters and conserved CREs}

Estimating substitution rates at chaperone promoters in Cryotnotothenioidei and determining whether they have undergone accelerated evolution relative to other perciformes first required fitting different null substitution models to multiple sequence alignments of orthologous promoter regions input with the phylogenetic topography described earlier. This task was achieved using an HKY85 + Gap substitution model in the phlyoFit function of the evolutionary genomics software package PHAST [61], v1.5. The HKY85 + Gap model treats gaps as a fifth character. The decision to use this substitution model was made given (i) the prevalence of indels within multiple alignments generated in this study and (ii) the functional consequences indels can yield within proximal promoters $[90,91]$.

Multiple alignments of promoter regions and their corresponding null substitution models were used to test whether significantly accelerated mutation rates have occurred among chaperone promoters within the Cryonotothenioidei node relative to all other species examined. Treating promoters as single genomic features, this task was carried out in phyloP using the CONACC function, which simultaneously tests for significant conservation or acceleration using base-by-base methods or genomic features. Test statistics for phyloP were derived from the SCORE method for detecting selection, which is described by Pollard et al. 2010 to be more statistically powerful in instances of weaker selection as opposed to similar methods such as likelihood ratio tests [62]. As strong selective forces were not expected to be acting on promoter features at large, the SCORE method was selected for this purpose. Using a base-by-base setting in phyloP set to the CONACC function and SCORE test, the proportion of promoters regions under significant acceleration or conservation were determined using an FDR cut-off of $<0.1$. This was performed separately for Cryonotothenioidei, subpolar notothens, and non-notothen perciformes in order to 
determine whether accelerated mutation rates in cryonotothen chaperone promoters occurred at a significantly greater number of bases than in other lineages using an ordinal logistic model.

A similar pipeline was used to test for accelerated mutation rates in Cryonotothenioidei relative to other fishes at highly-conserved bases and CREs. First, multiple alignments of promoter regions and their corresponding null substitution models were input to phastCons [61] in order to generate base-by-base conservation scores as well as predictions for highly-conserved CRE features. phastCons was run using the following parameters: $\rho=$ 0.4 ; target coverage $=0.3$; expected length $=45$. An ordinal logistic model was used to test whether the proportion of significantly accelerated bases predicted by phyloP varied as a function of conservation (i.e. phastCons score), the gene family of a promoter, and the interaction of both predictors. Lastly, phyloP was used to test for significant acceleration among cryonotothens and subpolar notothens at conserved CREs identified via phastCons using likelihood ratio tests (LRTs) rather than SCORE tests in light of LRTs similarities to the SCORE test and greater statistical power in detecting selection at smaller genomic features [62]. This process was repeated for a subset of the examined phylogeny that focused on Notothenioidei alone, for which a separate null substitution model was fitted in phyloP using the same settings described earlier and the same input multiple sequence alignments. This was done in order to identify CREs that were conserved among Notothenioidei, but not necessarily Perciformes, that exhibited accelerated rates of mutation in later lineages.

\section{Detection of CRE gains and losses across evolution}

Using the dless function of the PHAST software package set to parameters identical to those described for phastCons, gains and losses of conserved non-coding elements were identified within chaperone promoters across (i) the full phylogeny of 32 species included in this study fitted to corresponding null substitution models and (ii) the Notothenioidei subset of this phylogeny fitted to corresponding null substitution models for notothens alone. Null substitution models employed in the dless function were identical to those fitted for use in phyloP.

\section{Supplementary information}

Supplementary information accompanies this paper at https://doi.org/10 1186/s12862-019-1524-y.

Additional file 1: Links and accessions for fish genome assemblies and chaperone CDS.

Additional file 2. Mean HSE counts and densities per species among chaperone promoters.
Additional file 3. CONACC output of base-by-base conservation/ acceleration scores and p-values for each gene and clade. bp number ascends in 5' - 3' order across promoters toward 1st exons.

Additional file 4. phastCons scores across chaperone promoters derived from (i) a substitution model fitted to all species and (ii) a substitution model fitted to Notothenioidei. bp numbers negatively descend in $3^{\prime}-5^{\prime}$ order across promoters beginning at $5^{\prime}$ end of 1 st exons.

\section{Abbreviations}

CDS: Coding sequence; CRE: cis-regulatory element; CV: Coefficient of variation; HSE: Heat shock element; LRT: Likelihood ratio test

\section{Acknowledgements}

We thank Dr. Daniel Crocker and Dr. Nathaniel Rank (Sonoma State University) for their input on early versions of the manuscript and guidance relating to statistical analyses. We also thank Dr. Lars Tomanek (California Polytechnic State University, San Luis Obispo) for his insight regarding the cis-regulation of heat shock genes and Katherine Corn (University of

California, Davis) for her suggestions regarding phylogenetic correction. We would also like to thank our editor and three anonymous reviewers for their suggestions which materially improved the manuscript.

\section{Authors' contributions}

SPP conceived the scope of the study, advised SNB throughout its completion, and edited the manuscript. SNB conceived the approach and specific aims of the study, carried out each analysis, and wrote and edited the manuscript. Both authors have read and approved the manuscript.

\section{Funding}

This work was supported by the National Science Foundation in the form of research grants (PLR 1447291) awarded to SPP and a CSU COAST Graduate Student Research Award to SNB. Both awards provided funds for graduate student support during this project.

\section{Availability of data and materials}

All data generated or analyzed during this study are cited or included in this article and its supplementary information files.

\section{Competing interests}

The authors declare that they have no competing interests.

Received: 15 May 2019 Accepted: 1 October 2019

Published online: 06 November 2019

\section{References}

1. Rockman MV, Wray GA. Abundant raw material for cis-regulatory evolution in humans. Mol Biol Evol. 2002;19:1991-2004

2. Moses AM, Pollard DA, Nix DA, lyer VN, Li XY, Biggin MD, Eisen MB. Largescale turnover of functional transcription factor binding sites in Drosophila. PLoS Comput Biol. 2006;2:e130.

3. Borneman AR, Gianoulis TA, Zhang ZD, Yu H, Rozowsky J, Seringhaus MR, Wang LY, Gerstein M, Michael S. Divergence of transcription factor binding sites across related yeast species. Science. 2007;317:815-9.

4. Doniger SW, Fay JC. Frequent gain and loss of functional transcription factor binding sites. PLoS Comput Biol. 2007;3:e99.

5. Bradley RK, Li XY, Trapnell C, Davidson S, Pachter L, Chu HC, Tonkin LA Biggin MD, Eisen MB. Binding site turnover produces pervasive quantitative changes in transcription factor binding between closely related Drosophila species. PLoS Biol. 2010;8:e1000343.

6. Rockman MV, Hahn MW, Soranzo N, Zimprich F, Goldstein DB, Wray GA. Ancient and recent positive selection transformed opioid cis-regulation in humans. PLoS Biol. 2005;3:e387

7. Danko CG, Choate LA, Marks BA, Rice EJ, Wang Z, Chu T, Martins AL, Dukler $\mathrm{N}$, Coonrod SA, Tait Wojno ED, et al. Dynamic evolution of regulatory element ensembles in primate CD4(+) T cells. Nat Ecol Evol. 2018;2:537-48.

8. Prabhakar S, Visel A, Akiyama JA, Shoukry M, Lewis KD, Holt A, Plajzer-Frick I, Morrison $\mathrm{H}$, Fitzpatrick DR, Afzal $\mathrm{V}$, et al. Human-specific gain of function in a developmental enhancer. Science. 2008;321:1346-50. 
9. Rebeiz M, Jikomes N, Kassner VA, Carroll SB. Evolutionary origin of a novel gene expression pattern through co-option of the latent activities of existing regulatory sequences. Proc Natl Acad Sci U S A. 2011;108:10036-43.

10. Rogers WA, Salomone JR, Tacy DJ, Camino EM, Davis KA, Rebeiz M, Williams TM. Recurrent modification of a conserved cis-regulatory element underlies fruit fly pigmentation diversity. PLoS Genet. 2013;9:e1003740.

11. Koshikawa S, Giorgianni MW, Vaccaro K, Kassner VA, Yoder JH, Werner T, Carroll SB. Gain of cis-regulatory activities underlies novel domains of wingless gene expression in Drosophila. Proc Natl Acad Sci U S A. 2015;112: 7524-9.

12. Villar D, Berthelot C, Aldridge S, Rayner TF, Lukk M, Pignatelli M, Park TJ, Deaville R, Erichsen JT, Jasinska AJ, et al. Enhancer evolution across 20 mammalian species. Cell. 2015;160:554-66.

13. Boyd JL, Skove SL, Rouanet JP, Pilaz L, Bepler T, Gordan R, Wray GA, Silver DL. Human-chimpanzee differences in a FZD8 enhancer alter cell-cycle dynamics in the developing neocortex. Curr Biol. 2015;25:772-9.

14. Bulger M, Groudine M. Functional and mechanistic diversity of distal transcription enhancers. Cell. 2011;144:327-39.

15. Maeso I, Acemel RD, Gomez-Skarmeta JL. Cis-regulatory landscapes in development and evolution. Curr Opin Genet Dev. 2017:43:17-22.

16. Signor SA, Nuzhdin SV. The evolution of gene expression in cis and trans. Trends Genet. 2018;34:532-44.

17. Olivera BM, Watkins $M$, Bandyopadhyay $P$, Imperial JS, de la Cotera EP, Aguilar MB, Vera EL, Concepcion GP, Lluisma A. Adaptive radiation of venomous marine snail lineages and the accelerated evolution of venom peptide genes. Ann N Y Acad Sci. 2012;1267:61-70.

18. Pelosi L, Kuhn L, Guetta D, Garin J, Geiselmann J, Lenski RE, Schneider D. Parallel changes in global protein profiles during long-term experimental evolution in Escherichia coli. Genetics. 2006;173:1851-69.

19. Dong Y, Miller LP, Sanders JG, Somero GN. Heat-shock protein 80 (Hgsp70) expression in four limpets of the genus Lottia: interspecific variation in constitutive and inducible synthesis correlates with in situ exposure to heat stress. Biol Bull. 2008;215:173-81.

20. Latta LC, Weider $\sqcup$, Colbourne JK, Pfrender ME. The evolution of salinity tolerance in Daphnia: a functional genomics approach. Ecol Lett. 2012;15:794-802.

21. Barshis DJ, Ladner JT, Oliver TA, Seneca FO, Traylor-Knowles N, Palumbi SR. Genomic basis for coral resilience to climate change. Proc Natl Acad Sci U S A. 2013;110:1387-92

22. Haguenauer A, Zuberer F, Ledoux JB, Aurelle D. Adaptive abilities of the Mediterranean red coral Corallium rubrum in a heterogeneous and changing environment: from population to functional genetics. J Exp Mar Biol Ecol. 2013:449:349-57.

23. Kenkel CD, Meyer E, Matz MV. Gene expression under chronic heat stress in populations of the mustard hill coral (Porites astreoides) from different thermal environments. Mol Ecol. 2013;22:4322-34.

24. Franssen SU, Gu J, Winters G, Huylmans AK, Wienpahl I, Sparwel M, Coyer JA, Olsen JL, Reusch TB, Bornberg-Bauer E. Genome-wide transcriptomic responses of the seagrasses Zostera marina and Nanozostera noltii under a simulated heatwave confirm functional types. Mar Genomics. 2014;15:65-73.

25. Gleason LU, Burton RS. RNA-seq reveals regional differences in transcriptome response to heat stress in the marine snail Chlorostoma funebralis. Mol Ecol. 2015;24:610-27.

26. Poley JD, Igboeli OO, Fast MD. Towards a consensus: multiple experiments provide evidence for constitutive expression differences among sexes and populations of sea lice (Lepeophtheirus salmonis) related to emamectin benzoate resistance. Aquaculture. 2015:448:445-50.

27. Kenkel CD, Matz MV. Gene expression plasticity as a mechanism of coral adaptation to a variable environment. Nat Ecol Evol. 2016;1:14.

28. Lima TG, Willett CS. Locally adapted populations of a copepod can evolve different gene expression patterns under the same environmental pressures. Ecol Evol. 2017;7:4312-25.

29. Wright RM, Kenkel CD, Dunn CE, Shilling EN, Bay LK, Matz MV. Intraspecific differences in molecular stress responses and coral pathobiome contribute to mortality under bacterial challenge in Acropora millepora. Sci Rep. 2017;7:2609.

30. Avivi A, Resnick MB, Nevo E, Joel A, Levy AP. Adaptive hypoxic tolerance in the subterranean mole rat Spalax ehrenbergi: the role of vascular endothelial growth factor. FEBS Lett. 1999;452:133-40.

31. Tomanek L, Somero GN. Evolutionary and acclimation-induced variation in the heat-shock responses of congeneric marine snails (genus Tegula) from different thermal habitats: implications for limits of thermotolerance and biogeography. J Exp Biol. 1999;202:2925-36.
32. Shams I, Avivi A, Nevo E. Oxygen and carbon dioxide fluctuations in burrows of subterranean blind mole rats indicate tolerance to hypoxichypercapnic stresses. Comp Biochem Physiol A Mol Integr Physiol. 2005;142:376-82.

33. Lampe RH, Cohen NR, Ellis KA, Bruland KW, Maldonado MT, Peterson TD, Till CP, Brzezinski MA, Bargu S, Thamatrakoln K, et al. Divergent gene expression among phytoplankton taxa in response to upwelling. Environ Microbiol. 2018;20:3069-82.

34. Franzellitti S, Airi V, Calbucci D, Caroselli E, Prada F, Voolstra CR, Mass T, Falini G, Fabbri E, Goffredo S. Transcriptional response of the heat shock gene hsp70 aligns with differences in stress susceptibility of shallow-water corals from the Mediterranean Sea. Mar Environ Res. 2018;140:444-54.

35. Gatenby RA, Gillies RJ. Glycolysis in cancer: a potential target for therapy. Int J Biochem Cell Biol. 2007:39:1358-66.

36. Caro I, Low JA. The role of the hedgehog signaling pathway in the development of basal cell carcinoma and opportunities for treatment. Clin Cancer Res. 2010;16:3335-9.

37. Akakura N, Kobayashi M, Horiuchi I, Suzuki A, Wang J, Chen J, Niizeki H, Kawamura K, Hosokawa M, Asaka M. Constitutive expression of hypoxiainducible factor-1 renders pancreatic cancer cells resistant to apoptosis induced by hypoxia and nutrient deprivation. Cancer Res. 2001;61:6548-54.

38. Morschhauser J. The development of fluconazole resistance in Candida albicans - an example of microevolution of a fungal pathogen. J Microbiol. 2016:54:192-201.

39. Heilmann CJ, Schneider S, Barker KS, Rogers PD, Morschhauser J. An A643T mutation in the transcription factor Upc2p causes constitutive ERG11 upregulation and increased fluconazole resistance in Candida albicans. Antimicrob Agents Chemother. 2010;54:353-9.

40. Dunkel N, Liu TT, Barker KS, Homayouni R, Morschhauser J, Rogers PD. A gain-of-function mutation in the transcription factor Upc2p causes upregulation of ergosterol biosynthesis genes and increased fluconazole resistance in a clinical Candida albicans isolate. Eukaryot Cell. 2008;7:1180-90.

41. Scoville AG, Pfrender ME. Phenotypic plasticity facilitates recurrent rapid adaptation to introduced predators. Proc Natl Acad Sci U S A. 2010;107: 4260-3.

42. Velotta JP, Ivy CM, Wolf CJ, Scott GR, Cheviron ZA. Maladaptive phenotypic plasticity in cardiac muscle growth is suppressed in high-altitude deer mice. Evolution. 2018;72:2712-27.

43. Hofmann GE, Buckley BA, Airaksinen S, Keen JE, Somero GN. Heat-shock protein expression is absent in the Antarctic fish Trematomus bernacchii (family Nototheniidae). J Exp Biol. 2000;203:2331-9.

44. Place SP, Hofmann GE. Constitutive expression of a stress-inducible heat shock protein gene, hsp70, in phylogenetically distant Antarctic fish. Polar Biol. 2004;28:261-7.

45. Clark MS, Fraser KPP, Burns G, Peck LS. The HSP70 heat shock response in the Antarctic fish Harpagifer antarcticus. Polar Biol. 2007:31:171-80

46. La Terza AM, C.; Luporini P. Divergence between two Antarctic species of the ciliate Euplotes, E. focardii and E. nobilii, in the expression of heat-shock protein 70 genes. Mol Ecol. 2001;10:1061-7.

47. Todgham AE, Hoaglund EA, Hofmann GE. Is cold the new hot? Elevated ubiquitin-conjugated protein levels in tissues of Antarctic fish as evidence for cold-denaturation of proteins in vivo. J Comp Physiol B. 2007;177:85766.

48. Bilyk KT, Vargas-Chacoff L, Cheng CC. Evolution in chronic cold: varied loss of cellular response to heat in Antarctic notothenioid fish. BMC Evol Biol. 2018;18:143.

49. Teigen L. Induction of heat shock response proteins in cold-adapted and cold-acclimated fishes. Fairbanks: University of Alaska Fairbanks; 2014.

50. Hofmann GE, Lund SG, Place SP, Whitmer AC. Some like it hot, some like it cold: the heat shock response is found in New Zealand but not Antarctic notothenioid fishes. J Exp Mar Biol Ecol. 2005;316:79-89.

51. Shin SC, Ahn DH, Kim SJ, Pyo CW, Lee H, Kim M, Lee J, Lee JE, Detrich HW 3rd, Postlethwait JH, et al. The genome sequence of the Antarctic bullhead notothen reveals evolutionary adaptations to a cold environment. Genom Biol. 2014;15:468.

52. Enzor LA, Place SP. Is warmer better? Decreased oxidative damage in notothenioid fish after long-term acclimation to multiple stressors. J Exp Biol. 2014:217:3301-10.

53. Huth TJ, Place SP. RNA-seq reveals a diminished acclimation response to the combined effects of ocean acidification and elevated seawater temperature in Pagothenia borchgrevinki. Mar Genomics. 2016;28:87-97. 
54. Huth TJ, Place SP. Transcriptome wide analyses reveal a sustained cellular stress response in the gill tissue of Trematomus bernacchii after acclimation to multiple stressors. BMC Genomics. 2016;17:127.

55. Lindquist S. The heat shock response. Annu Rev Biochem. 1986:55:1151-91.

56. Brawand D, Wagner CE, Li Yl, Malinsky M, Keller I, Fan S, Simakov O, Ng AY,

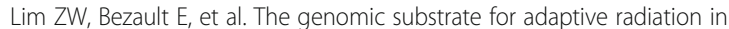
African cichlid fish. Nature. 2014;513:375-81.

57. Chen B, Feder ME, Kang L. Evolution of heat-shock protein expression underlying adaptive responses to environmental stress. Mol Ecol. 2018;27: 3040-54.

58. Tian S, Haney RA, Feder ME. Phylogeny disambiguates the evolution of heat-shock cis-regulatory elements in Drosophila. PLoS One. 2010;5:e10669.

59. Nguyen AD, Gotelli NJ, Cahan SH. The evolution of heat shock protein sequences, cis-regulatory elements, and expression profiles in the eusocial hymenoptera. BMC Evol Biol. 2016;16:15.

60. Tangwancharoen S, Moy GW, Burton RS. Multiple modes of adaptation: regulatory and structural evolution in a small heat shock protein gene. Mol Biol Evol. 2018;35:2110-9.

61. Hubisz MJ, Pollard KS, Siepel A. PHAST and RPHAST: phylogenetic analysis with space/time models. Brief Bioinform. 2011;12:41-51.

62. Pollard KS, Hubisz MJ, Rosenbloom KR, Siepel A. Detection of nonneutral substitution rates on mammalian phylogenies. Genome Res. 2010;20:110-21.

63. Pelham HRBB. Mariann: a synthetic heat-shock promoter element confers heat-inducibility on the herpes simplex virus thymidine kinase gene. EMBO J. 1982;1:1473-7.

64. Amin J, Ananthan J, Voellmy R. Key features of heat shock regulatory elements. Mol Cell Biol. 1988:8:3761-9.

65. Hoffmann RD, Palmgren M. Purifying selection acts on coding and noncoding sequences of paralogous genes in Arabidopsis thaliana. BMC Genomics. 2016;17:456

66. Asthana S, Noble WS, Kryukov G, Grant CE, Sunyaev S, Stamatoyannopoulos $J$ A. Widely distributed noncoding purifying selection in the human genome Proc Natl Acad Sci U S A. 2007;104:12410-5

67. Arnold CD, Gerlach D, Spies D, Matts JA, Sytnikova YA, Pagani M, Lau NC, Stark A. Quantitative genome-wide enhancer activity maps for five Drosophila species show functional enhancer conservation and turnover during cis-regulatory evolution. Nat Genet. 2014;46:685-92.

68. de Souza FS, Franchini LF, Rubinstein M. Exaptation of transposable elements into novel cis-regulatory elements: is the evidence always strong? Mol Biol Evol. 2013;30:1239-51.

69. Jareborg N, Birney E, Durbin R. Comparative analysis of noncoding regions of 77 orthologous mouse and human gene pairs. Genome Res. 1999;9:815-24.

70. Cotney J, Leng J, Yin J, Reilly SK, DeMare LE, Emera D, Ayoub AE, Rakic P, Noonan JP. The evolution of lineage-specific regulatory activities in the human embryonic limb. Cell. 2013;154:185-96.

71. Cheng Y, Ma Z, Kim BH, Wu W, Cayting P, Boyle AP, Sundaram V, Xing X, Dogan $\mathrm{N}$, Li J, et al. Principles of regulatory information conservation between mouse and human. Nature. 2014;515:371-5.

72. Barrier M, Robichaux RH, Purugganan MD. Accelerated regulatory gene evolution in an adaptive radiation. Proc Natl Acad Sci U S A. 2001;98: 10208-13.

73. Near TJ, MacGuigan DJ, Parker E, Struthers CD, Jones CD, Dornburg A. Phylogenetic analysis of Antarctic notothenioids illuminates the utility of RADseq for resolving Cenozoic adaptive radiations. Mol Phylogenet Evol. 2018:129:268-79.

74. Daane JM, Dornburg A, Smits P, MacGuigan DJ, Brent Hawkins M, Near TJ, William Detrich lii $\mathrm{H}$, Harris MP. Historical contingency shapes adaptive radiation in Antarctic fishes. Nat Ecol Evol. 2019;3:1102-9.

75. Ahn DH, Shin SC, Kim BM, Kang S, Kim JH, Ahn I, Park J, Park H. Draft genome of the Antarctic dragonfish, Parachaenichthys charcoti. Gigascience. 2017;6:1-6.

76. Baalsrud HT, Torresen OK, Hongro Solbakken M, Salzburger W, Hanel R, Jakobsen KS, Jentoft S. De novo gene evolution of antifreeze glycoproteins in codfishes revealed by whole genome sequence data. Mol Biol Evol. 2017.

77. Kim BM, Amores A, Kang S, Ahn DH, Kim JH, Kim IC, Lee JH, Lee SG, Lee H, Lee J, et al. Antarctic blackfin icefish genome reveals adaptations to extreme environments. Nat Ecol Evol. 2019:3:469-78.

78. Chen L, Lu Y, Li W, Ren Y, Yu M, Jiang S, Fu Y, Wang J, Peng S, Bilyk KT, et al. The genomic basis for colonizing the freezing Southern Ocean revealed by Antarctic toothfish and Patagonian robalo genomes. Gigascience. 2019;8:giz016.
79. Haussler D, O'Brien SJ, Ryder OA, Barker FK, Clamp M, Crawford AJ, Hanner R, Hanotte O, Johnson WE, McGuire JA, et al. Genome 10K: a proposal to obtain whole-genome sequence for 10000 vertebrate species. J Hered. 2009;100:659-74

80. Kasahara M, Naruse K, Sasaki S, Nakatani Y, Qu W, Ahsan B, Yamada T, Nagayasu Y, Doi K, Kasai Y, et al. The medaka draft genome and insights into vertebrate genome evolution. Nature. 2007:447:714-9.

81. Kai W, Kikuchi K, Tohari S, Chew AK, Tay A, Fujiwara A, Hosoya S, Suetake H, Naruse K, Brenner S, et al. Integration of the genetic map and genome assembly of fugu facilitates insights into distinct features of genome evolution in teleosts and mammals. Genome Biol Evol. 2011:3:424-42.

82. Zhang Z, Schwartz S, Wagner L, Miller W. A greedy algorithm for aligning DNA sequences. J Comput Biol. 2000;7:203-14.

83. Ozretic P, Bisio A, Musani V, Trnski D, Sabol M, Levanat S, Inga A. Regulation of human PTCH1b expression by different 5' untranslated region cisregulatory elements. RNA Biol. 2015;12:290-304.

84. Sievers F, Higgins DG. Clustal omega. Curr Protoc Bioinformatics. 2014;48:3. 13:11-6.

85. Grant CE, Bailey TL, Noble WS. FIMO: scanning for occurrences of a given motif. Bioinformatics. 2011;27:1017-8.

86. Revell L. Phytools: an R package for phylogenetic comparative biology (and other things). Methods Ecol Evol. 2012:3:217-23.

87. Chang J, Rabosky DL, Smith SA, Alfaro ME, Silvestro D. An R package and online resource for macroevolutionary studies using the ray-finned fish tree of life. Methods Ecol Evol. 2019;10:1118-24.

88. Torchiano M: effsize: Efficient effect size computation. In., Version 0.7.1 edn; 2019: R package.

89. Ho J, Tumkaya T, Aryal S, Choi H, Claridge-Chang A. Moving beyond P values: data analysis with estimation graphics. Nat Methods. 2019;16:565-6.

90. Xu Y, Shi T, Zhou Y, Liu M, Klaus S, Lan X, Lei C, Chen H. A novel PAX7 10bp indel variant modulates promoter activity, gene expression and contributes to different phenotypes of Chinese cattle. Sci Rep. 2018:8:1724.

91. Warren ST, Zhang F, Licameli GR, Peters JF. The fragile $X$ site in somatic cell hybrids: an approach for molecular cloning of fragile sites. Science. 1987; 237:420-3.

92. Vasadia DJ, Zippay ML, Place SP. Characterization of thermally sensitive miRNAs reveals a central role of the FoxO signaling pathway in regulating the cellular stress response of an extreme stenotherm, Trematomus bernacchii. Mar Genomics. 2019:100698.

93. Buckley BA, Place SP, Hofmann GE. Regulation of heat shock genes in isolated hepatocytes from an Antarctic fish, Trematomus bernacchii. J Exp Biol. 2004:207:3649-56.

\section{Publisher's Note}

Springer Nature remains neutral with regard to jurisdictional claims in published maps and institutional affiliations.
Ready to submit your research? Choose BMC and benefit from:

- fast, convenient online submission

- thorough peer review by experienced researchers in your field

- rapid publication on acceptance

- support for research data, including large and complex data types

- gold Open Access which fosters wider collaboration and increased citations

- maximum visibility for your research: over $100 \mathrm{M}$ website views per year

At BMC, research is always in progress.

Learn more biomedcentral.com/submission 